\title{
Flow-Driven Branching in a Frangible Porous Medium
}

\author{
Nicholas J. Derr®, ${ }^{1}$ David C. Fronk $\odot,{ }^{2}$ Christoph A. Weber, ${ }^{3}$ Amala Mahadevan $\odot,{ }^{4}$ \\ Chris H. Rycroft, ${ }^{1,5}$ and L. Mahadevan $\odot^{1,2,6, *}$ \\ ${ }^{1}$ John A. Paulson School of Engineering and Applied Sciences, Harvard University, Cambridge, Massachusetts 02138, USA \\ ${ }^{2}$ Department of Organismic and Evolutionary Biology, Harvard University, Cambridge, Massachusetts 02138, USA \\ ${ }^{3}$ Max Planck Institute for the Physics of Complex Systems, Dresden 01187, Germany \\ ${ }^{4}$ Woods Hole Oceanographic Institution, Woods Hole, Massachusetts 02450, USA \\ ${ }^{5}$ Computational Research Division, Lawrence Berkeley National Laboratory, Berkeley, California 94720, USA \\ ${ }^{6}$ Department of Physics, Harvard University, Cambridge, Massachusetts 02138, USA
}

(Received 21 June 2020; accepted 18 August 2020; published 6 October 2020)

\begin{abstract}
Channel formation and branching is widely seen in physical systems where movement of fluid through a porous structure causes the spatiotemporal evolution of the medium. We provide a simple theoretical framework that embodies this feedback mechanism in a multiphase model for flow through a frangible porous medium with a dynamic permeability. Numerical simulations of the model show the emergence of branched networks whose topology is determined by the geometry of external flow forcing. This allows us to delineate the conditions under which splitting and/or coalescing branched network formation is favored, with potential implications for both understanding and controlling branching in soft frangible media.
\end{abstract}

DOI: 10.1103/PhysRevLett.125.158002

Branching patterns in porous media are common in many natural settings that include both living and nonliving matter [1]. The formation of arborized patterns in physical and chemical systems is driven by a variety of processes all of which involve a combination of erosion, transport, and deposition. On the laboratory scale, these processes can involve chemical dissolution of brittle matrices by a penetrating reactive fluid $[2,3]$, advective rearrangement of unconsolidated media, dielectric breakdown of conducting media [4,5], formation of fingerlike protrusions in dense granular suspensions [6], formation of beach rills in natural drainage systems [7,8], etc. On planetary scales, melt transport in the mantle arises via branching morphologies that lead to localized channels of widths up to $100 \mathrm{~m}$ [9-11], and water-driven erosion and branching in glaciers arises on scales of the order of $10 \mathrm{~m}$ [12]. In biological systems, the best known arborized systems are vasculatures in plants and animals. These arise through morphogenetic mechanisms involving gradients and physical flows that arrange and rearrange matter through a variety of feedback mechanisms at the cellular, organismal, and societal level $[13,14]$. Examples include slime molds [15], vascular networks [16], and nest architectures of social insects [17].

Models based on porous flow theory $[18,19]$ are capable of describing flow through these branched networks. However, their formation requires nonlinear models with multiple evolving phase boundaries which are still only partially understood both theoretically and experimentally. Here, we propose a simple model via an effective continuum theory that links flow, permeability, and pressure gradients by considering pore-scale grain dislodgement in a relatively brittle structure. Numerical solutions of the resulting governing equations show the emergence of branching morphologies through selective erosion and subsequent flow enhancement.

Mathematical model.-Our starting point is a fluid-filled porous domain $\Omega$ comprised of a rigid grain microstructure with characteristic pore size $l$, as in the Fig. 1(a) inset. The fluid is of viscosity $\eta$ and density $\rho$. On length scales large compared to the pore size $L \gg l$, we can define macroscopic continuum fields that include the solid fraction $\phi(\mathbf{x}, t)$ and volumetric fluid flux $\mathbf{q}(\mathbf{x}, t)$ as averages of microscopic quantities [20]. Pressure gradients over macroscopic lengths $\Gamma \sim|\nabla p|$ drive motion of the interstitial fluid at velocities $V \sim|\mathbf{q}|$ relative to the pore structure. Balancing the pressure gradients and viscous resistance at a scaling level implies that $\Gamma \sim \eta V / l^{2}$, so that individual grains feel forces of magnitude $\eta V l \sim \Gamma l^{3}$. When these overcome the attractive forces providing microstructural integrity, grains are dislodged, and the local permeability of the medium evolves. Symmetry arguments introduced in [21] suggest an erosion rate depending on the pressure gradient magnitude as a proxy for pore-scale grain tractions. Denoting the network breaking stress $B(\mathbf{x}, t)$, we write the most general such rate law

$$
\partial_{t} \phi=-e_{0} \phi f(|\nabla p|, B / l)
$$

where $e_{0}$ is an erosion rate and $f$ is a nonnegative dimensionless function that vanishes for $|\nabla p|<B / l$. Our original model [21] accounted for the relative motion 

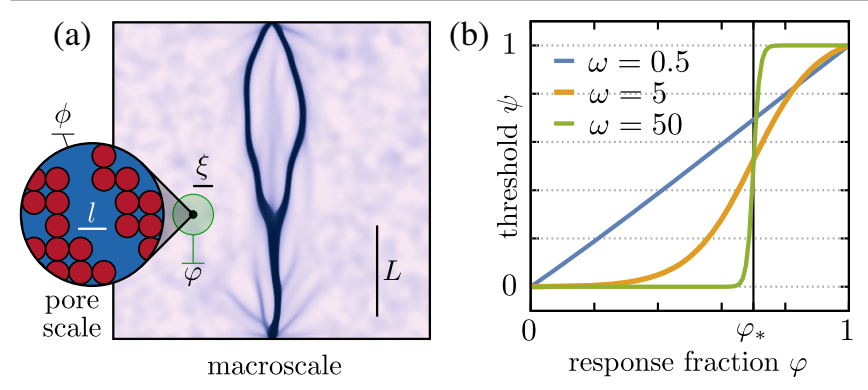

FIG. 1. Schematic of model fields, length scales, and erosion criteria. (a) Branched patterns in porous media can emerge on macroscopic lengths $L$ due to interactions at the pore size $l$. In this simulated pattern, eroded regions of low solid fraction $\phi(\mathbf{x}, t)$ are blue. At a given point on the macroscale (black dot), $\phi$ is the fraction of a pore-scale integration volume (inset) occupied by rigid grain microstructure (red circles). Fluid-mediated forces on grains induce stresses over a macroscopic region (shaded green circle) characterized by the communication length $\xi$. The response fraction $\varphi$ is the spatial average of $\phi$ throughout this region. (b) The erosion threshold function $\psi(\varphi)=$ $c_{0} \tanh \left[\omega\left(\varphi-\varphi_{*}\right)\right]+c_{1} \in[0,1]$ represents resistance to grain dislodgement at response fraction $\varphi$.

of the grains, fluid, and static porous medium via a threephase description allowing dislodged grains to deposit back onto the microstructure. Here, we omit deposition and focus on a simpler two-phase model assuming loose grains to be indistinguishable from fluid.

In terms of a characteristic breaking stress $B_{0}$ and time scale $\tau=1 / e_{0}$, we can define a characteristic length $L=$ $l\left(B_{0} / \eta e_{0}\right)$ and pressure gradient magnitude $\Gamma=B_{0} / l$. This allows us to rescale our variables and parameters accordingly; they should be assumed dimensionless for the remainder of the Letter unless otherwise specified. (Please see Supplemental Material (SM) section SM.1 [22] for the full dimensional system of equations.)

Assuming that the solid is relatively stiff but brittle so that it does not deform, the volumetric fluid flux $\mathbf{q}$ is well described by Darcy's law

$$
\mathbf{q}=-\kappa(\phi) \boldsymbol{\nabla} p, \quad \kappa(\phi)=\frac{(1-\phi)^{3}}{\phi^{2}},
$$

where the dimensionless permeability $\kappa(\phi)$ is the wellknown Carman-Kozeny relation [18,19]. Furthermore, if the fluid is incompressible, conservation of mass implies

$$
\boldsymbol{\nabla} \cdot \mathbf{q}=-s(\mathbf{x}, t),
$$

where $s(\mathbf{x}, t)$ is the rate at which fluid is depleted due to processes such as bulk reaction or evaporation. By combining the previous two equations, $\mathbf{q}$ can be eliminated to obtain an elliptic equation for the pressure,

$$
\boldsymbol{\nabla} \cdot[\kappa(\phi) \nabla p]=s .
$$

Boundary conditions correspond to specified fluxes $q_{\text {in }}$ and $q_{\text {out }}$ on boundary regions of inflow $\partial \Omega_{\text {in }}$ and outflow $\partial \Omega_{\text {out }}$ (see SM.2 [22] for details). We note the flow direction, which is determined by the boundary flux and fluid depletion signs, may be reversed with no change to morphogenic pattern formation because the erosion rule (1) is agnostic to the substitution $\boldsymbol{\nabla} p \rightarrow-\nabla p$.

To close the system, we must relate the dimensionless erosion rate $f$ to the fields $\phi(\mathbf{x}, t)$ and $p(\mathbf{x}, t)$. A minimal analytic form for $f$ suggests $f=\max \{0, \boldsymbol{\nabla} p \cdot \nabla p-$ $\left.B^{2} / l^{2}\right\}$. The breaking stress $B(\mathbf{x}, t)$ is itself is a nonlocal function of the solid fraction, depending on the grain density within a region of size $\xi$, a stress communication length which may depend on the porosity. Here, we assume the following hierarchy of constant lengths $l \ll \xi \ll L$, consistent with frangible brittle solids. In this limit, we introduce a simple erosion threshold $B^{2} / l^{2}=\psi(\varphi)$, defining the response fraction $\varphi(\mathbf{x}, t)$ as the convolution of $\phi(\mathbf{x}, t)$ with a Gaussian kernel of length scale $\xi$, representing a spatial average of the solid fraction as shown in Fig. 1(a) (see SM.3 [22] for details). Thus, the dimensionless form of the erosion rate law (1) becomes

$$
\partial_{t} \phi=-\phi \max \{0, \boldsymbol{\nabla} p \cdot \nabla p-\psi(\varphi)\} .
$$

For the functional form of the threshold, we consider a sigmoid $\psi(\varphi) \in[0,1]$ centered at a critical phase fraction $\varphi_{*}$, where the behavior is roughly linear over a scale $\Delta \varphi \sim 1 / \omega$, where $\omega$ represents a sharpness parameter as shown in Fig. 1(b). See SM.4 [22] for the exact form. We note that our functional choice satisfies $\psi^{\prime}(\varphi)>0$, i.e., the medium becomes more resistant to erosion at larger $\varphi$.

Equations (4) and (5) together determine the evolution of the permeability of the porous medium, $\phi(\mathbf{x}, t)$, and the pressure, $p(\mathbf{x}, t)$, once we specify an initial condition. Ignoring anisotropy in grain orientation and packing, we set $\phi(\mathbf{x}, 0)=\phi_{0}+\delta \phi(\mathbf{x})$, with $\phi_{0}$ a constant and $\delta \phi$ a perturbed packing structure described as a random Gaussian thermal noise field with zero mean, variance $\sigma_{\phi}^{2}$, and correlation length $\zeta \gg l$ satisfying

$$
\langle\delta \phi(\mathbf{x}) \delta \phi(\mathbf{y})\rangle_{r}=\sigma_{\phi}^{2} \exp (-r / \zeta) .
$$

Here, $\langle *\rangle_{r}=\int_{\Omega}(*) d \mathbf{x} d \mathbf{y} / \operatorname{vol}(\Omega)$ is a spatial average over all $\mathbf{x}, \mathbf{y} \in \Omega$ such that $|\mathbf{x}-\mathbf{y}|=r$.

The correlation length $\zeta$ and stress communication length $\xi$ control the characteristic channel width $w_{c}$. From (5), loss of solid material at a point reduces resistance to further erosion in a surrounding neighborhood of size $\xi$-qualitatively similar to descriptions of nonlocal damage accumulation in settings such as hydraulic fracturing [23]. Features in the $\phi$ field, initially of size $\zeta$, correspond to smoothed features in the $\varphi$ field. Thus, the channel width scale satisfies $\zeta^{2}<w_{c}^{2}<\xi^{2}+\zeta^{2}$, approaching the small limit for large values of the packing variance 
$\sigma_{\phi}^{2}$ and vice versa, consistent with results obtained using the three-phase model [21]. The width of a given channel scales with $w_{c}$ and varies with the amount of flux it conducts. See SM.5 [22] for details.

Before considering the spatiotemporal evolution of the flow and permeability, we examine the local dependence of erosion on the threshold shape $\psi(\varphi)$ and local flux q. Letting $\langle *\rangle=\int_{A}(*) d \mathbf{x} / \operatorname{vol}(A)$ denote a spatial average over a mesoscopic region $A$, we introduce the scalar fields $\Phi=\langle\phi\rangle, G^{2}=\langle\nabla p \cdot \nabla p\rangle$, and $Q^{2}=\langle\mathbf{q} \cdot \mathbf{q}\rangle$. We see they satisfy $Q=-\kappa(\Phi) G$, derived by averaging (2). Differentiating this relation and combining it with an averaged (5) yields a set of purely time-dependent equations describing trajectories through forcing-response phase space. For eroding states with $G^{2}>\psi(\Phi)$,

$$
\begin{aligned}
\frac{d \Phi}{d t} & =-\Phi\left(G^{2}-\psi\right), \\
\frac{d\left(G^{2}\right)}{d t} & =2 G^{2}\left[\left(\frac{\Phi \kappa^{\prime}}{\kappa}\right)\left(G^{2}-\psi\right)+\frac{\dot{Q}}{Q}\right] .
\end{aligned}
$$

Sustained erosion does not occur if $\dot{Q}=0$, for which points on the threshold surface $G^{2}=\psi$ are stable equilibria. Eroding states reach the threshold in finite time, as can be seen from (7a). For $\dot{Q} / Q>0$, this is not the case. The quantity $\Phi \kappa^{\prime} / \kappa<0$ is negative, so the squared gradient decays or grows when the first or second term in (7b), respectively, dominates the other. The majority of the system's evolution takes place along a monotonically increasing slow manifold $G_{s}(\Phi)$ where the two are balanced, corresponding to $d\left(G^{2}\right) / d t=0$. From (7b),

$$
G_{s}\left(G_{s}^{2}-\psi\right)=\frac{\dot{Q}}{\Phi \kappa^{\prime}},
$$

a cubic with one real root. In Fig. 2(a), we show the trajectories and slow manifolds for varying $\dot{Q}$. In Fig. 2(b), we plot the rate of erosion on the manifold, $f_{s}=G_{s}^{2}-\psi$, for thresholds of varying sharpness at a particular flux rate $\dot{Q}$. Theoretical bounds $f_{0}>f_{s}>f_{1}$, corresponding to constant thresholds $\psi=0$ and 1 , are plotted as black lines. Both are monotonically increasing, diverge as $\Phi \rightarrow 1$, and vanish as $\Phi \rightarrow 0$, so the rate of erosion slows over long times. This effect is mitigated by a transition from $f \approx f_{1}$ to $f_{0}$ near $\Phi \approx \varphi_{*}$. For sharp thresholds of large $\omega$, this effect is dominant and erosion accelerates upon reaching the transition region. The relative difference between the bounding rates, $\left(f_{0}-f_{1}\right) / f_{1}$, vanishes as $\Phi$ grows. In particular, when $\varphi<\varphi_{\text {star }}$ erosion is qualitatively faster than when $\varphi>\varphi_{\text {star. }}$. See SM.6 [22] for details.

Branching morphospaces.-Now, we turn to the spatiotemporal evolution of the flow and permeability fields in two-dimensional simulations. We aim to understand when, how, and what arborization motifs arise as a (a)



(b)

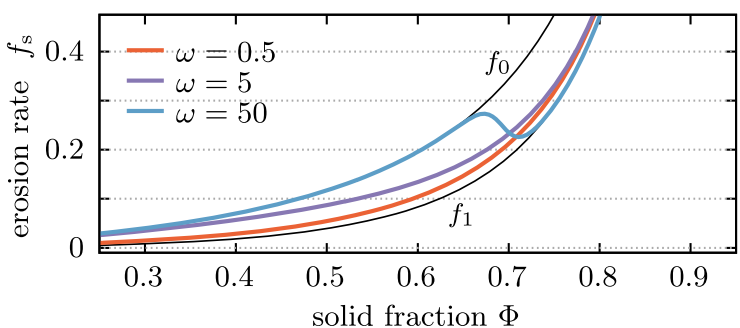

FIG. 2. Homogeneous model of erosion in the presence of an externally controlled flux $Q(t)$ defined by (7). (a) Phase trajectories through forcing-response space with initial condition $\left(\Phi, G^{2}\right)=(0.8,1.4)$ are shown for varying $\dot{Q}$. The erosion threshold $\psi(\Phi)$ has $\omega=8, \varphi_{*}=0.7$. Constant-flux trajectories reach the threshold quickly, stopping erosion in finite time. For $\dot{Q} \neq 0$, sustained erosion takes place at long times along the slow manifold $G_{s}^{2}(\Phi)$, plotted here as a translucent, thick line. (b) The erosion rate along the manifold, $f_{s}=G_{s}^{2}-\psi$, is plotted for three thresholds with $\varphi_{*}=0.7$ and varying sharpness $\omega$, subject to $\dot{Q}=0.1$. Bounds on the rate $f_{0}>f_{s}>f_{1}$ are plotted as black lines.

function of the boundary conditions, the dynamical rate of boundary fluxes, and the nature and functional form of the breaking threshold function. We integrate the coupled set of Eqs. (4) and (5) on a square domain $\Omega=[-5,5]^{2} \in \mathbb{R}^{2}$, employing a second-order forward Euler method with Richardson extrapolation for error estimation and adaptive time stepping [24]. See SM.7 [22] for details. We adopt boundary conditions which ramp up the flux from zero over a duration $T$. Introducing $r(t)=\min \{1, t / T\}$, we set the fluid depletion rate and boundary fluxes as $s(\mathbf{x}, t)=\hat{s} r(t), q_{\text {in }}(\mathbf{x}, t)=\hat{q}_{\text {in }} r(t)$, $q_{\text {out }}(\mathbf{x}, t)=\hat{q}_{\text {out }} r(t)$, where we have introduced a set of hatted constants corresponding to final magnitudes. This implies a uniform bulk fluid sink $\hat{s}$ evenly distributed throughout the domain. Similarly, the boundary fluxes are assumed to be uniform everywhere on the regions $\partial \Omega_{\text {in }}$ and $\partial \Omega_{\text {out }}$, which we center on the bottom and top walls of the domain, respectively.

There are two feedback mechanisms through which erosion in the model promotes itself. The first, observed in the homogeneous system, is the threshold reduction due to previous erosion. The second is a direct effect of the coupling between flux and permeability. According to (2), flux is preferentially directed along paths of larger permeability, so that, as it grows, flow from other parts of the domain is redistributed to eroded areas. In terms of the homogeneous phase space shown in Fig. 2(a), the resulting flux increase moves quickly eroding areas onto 


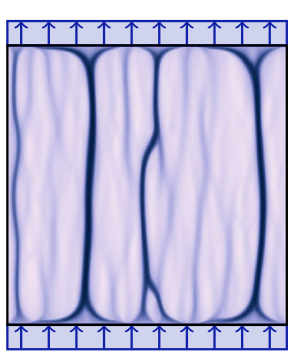

(a)

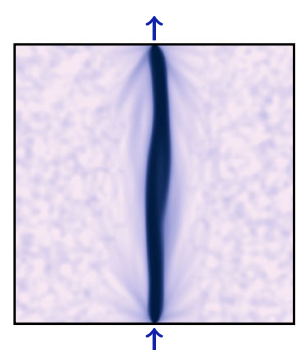

(b)

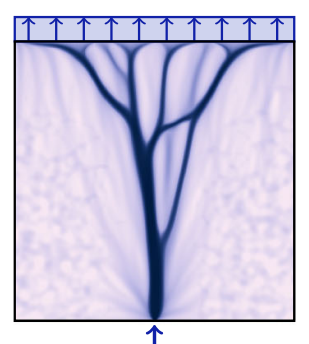

(c)

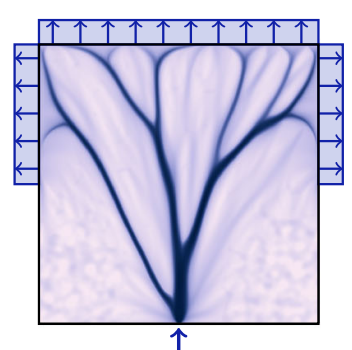

(d)



(e)

FIG. 3. Erosion patterns as functions of boundary conditions obtained by solving (4) and (5). The solid fraction $\phi(\mathbf{x}, t)$ is shown at $t=50$. The integrated flux through the system is ramped from zero to a final magnitude $F=\hat{q}_{\text {in }} w_{\text {in }}$ over a duration $T=10$. Flow enters on the bottom wall and exits through the top wall (a)-(d) or via evaporation in the bulk (e). If the regions of inflow and outflow are of similar size as in (a) and (b), flow is concentrated in straight channels. If they are of different sizes as in (c)-(e), one inflow channel branches into many at the outflow. The boundary widths satisfy $w_{\text {in }}=0.1$ or $10 ; w_{\text {out }}=0.1,10$ or 20 . Simulation parameters: grid size $1000^{2}, \phi_{0}=0.8, \varphi_{*}=0.7, \sigma_{\phi}=0.02, \zeta=0.1, \xi=0.1, \omega=6.5, F=0.8$. (See SM movie 1 [22] for visualizations of the dynamics of arborization that correspond to this figure.)

slow manifolds $G_{s}^{2}$ of higher $\dot{Q}$, speeding up erosion. Slowly eroding areas experience the opposite effect until so much flow is diverted that $\dot{Q} \leq 0$, so erosion ceases. In this way, flow enhancement leads directly to selective erosion of high- $\kappa$ channelized regions of width $w_{c}$. For a given integrated fluid flux at the boundary $F$, the number of channels to form in the absence of geometric constraints will scale as $N_{c} \sim F / w_{c}$; in what follows, $N_{c}>1$.

In Fig. 3, we show the results of simulations with four different combinations of boundary conditions and bulk evaporation rates. In the first four panels Figs. 3(a)-3(d), we set $\hat{s}=0$ and consider the effect of variation in boundary flux width. Generically, if either the inlet width $w_{\text {in }}$ or the outlet width $w_{\text {out }}$ are larger than the emergent channel size $w_{c}$, boundary fluxes induce the formation of multiple channels, as seen in Fig. 3(a). If both are less than $w_{c}$, a single channel is favored as in Fig. 3(b). (We note branching in these settings is possible-Fig. 1(a) shows a single channel split and consolidate-but only given conveniently located low- $\kappa$ regions of the initial condition in the $\xi \ll \zeta$ limit.) If neither is true, i.e., $w_{\text {in }}<w_{c}<w_{\text {out }}$, then $N_{c}$ channels are created at the outlet and one at the inlet, as in Figs. 3(c) and 3(d). In Fig. 3(e), we show the effect of bulk-evaporation driven flow with $\hat{s}>0$, a single inlet and no outlet. Because the channel width $w_{c} \ll L$ the system size, multiple channels form in the bulk, although their number and width is attenuated with distance from the inlet. These results may be summarized via a simple geometric argument suggesting a formula for reliable branch generation. If the number of channel heads distributed along the inlet and outlet are not the same, branching junctions arise in their linking, which is favored by flow continuity.

Finally, we consider the effects of varying the form of the erosion threshold function $\psi(\varphi)$, via its sharpness $\omega$, and the rate of flux increase, via the ramp-up time $T$. Figure 4 shows a grid of eroded patterns corresponding to combinations of these two parameters. Low rates of flux increase correspond to slow manifolds $G_{s}^{2}$ close to the threshold $\psi$, so small drops in the pressure gradient can yield $|\nabla p|^{2}<\psi$. Conversely, rapidly increasing fluxes induce large pressure gradients $|\nabla p|^{2} \gg \psi$ before flow reorganization can occur, leading to large-scale washout. We conclude that $T \gg 1$ is necessary for selective erosion. Increasing $\omega$ yields more

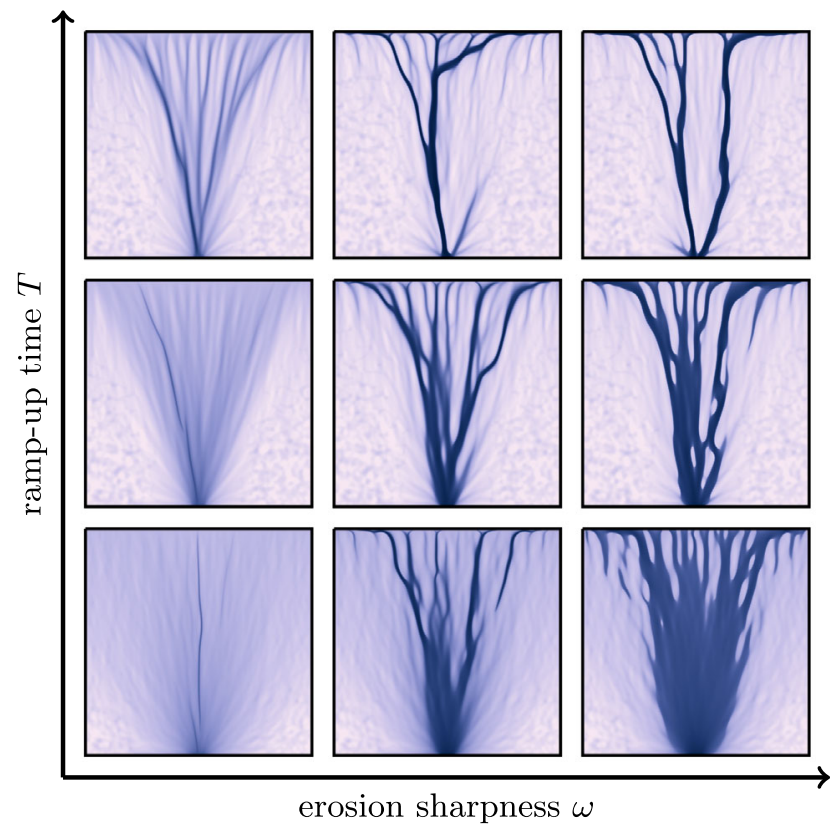

FIG. 4. Erosion patterns as functions of flux dynamics and threshold shape. The solid fraction $\phi(\mathbf{x}, t)$ is plotted with the Fig. 3 color scheme at $t=50$, subject to varied $\operatorname{ramp}$ time $T$ and sharpness $\omega$. Increasing $T$ or $\omega$ promotes confinement of erosion to a footprint which is smaller for more slowly increasing fluxes and larger for sharper thresholds. Boundary fluxes: $w_{\text {in }}=0.5$, $w_{\text {out }}=10, \hat{s}=0, \quad F=0.5$. Sharpness and ramp duration: $\omega=\{1,8,15\}, T=\{0,3,10\}$. Other parameters: grid size $1024^{2}, \phi_{0}=0.8, \varphi_{*}=0.7, \sigma_{\phi}=0.02, \zeta=0.08, \quad \xi=0.05$. (See SM movie 2 [22] for visualizations of the dynamics of arborization that correspond to this figure). 
erosion across the domain - consistent with the relationship between the erosion rate and $\omega$ as in Fig. 2(b) — and sharper boundaries between eroded and uneroded regions. This relates to qualitatively higher rates of erosion in the region $\varphi<\varphi_{*}$ corresponding to sharper thresholds. In physical terms, $\omega$ represents the immediacy with which a porous material's breaking stress vanishes after a critical amount of erosion. More selectivity in eroding below the critical solid fraction is associated with more rapidly vanishing breaking stresses.

These results are qualitatively similar to observations from the three-phase model [21], which predicted a spectrum of patterns from washout to defined channelization. Adding a mobile grain phase to this work would introduce another avenue for flow enhancement with an independent timescale associated with deposition. The regions of intermediate phase fraction on the left and bottom of Fig. 4 could redirect flow away as pores fill with grains, leading to the concentration of flow in the most-eroded regions and more defined patterns at long times.

Conclusions. - Our minimal continuum model for the coupled dynamics of erosion, flow, and permeability in a porous material shows how complex branching patterns can arise from simple causes. While the model and discussion are rooted in the language of frangible solids, our framework is broadly applicable beyond this setting, to branching patterns generated by local interactions subject to nonlocal flow constraints. Generalizing this to biological settings that feature nonlinear couplings such as that between nutrient concentration and flow behavior, e.g., if portions of solid may be flow-seeking or flow-avoiding [14] is a natural next step.

N.D. was partially supported by the NSF-Simons Center for Mathematical and Statistical Analysis of Biology at Harvard, Grant No. 1764269, and the Harvard Quantitative Biology Initiative. C. H. R. and N. D. were partially supported by the National Science Foundation under Grant No. DMS-1753203. C. H. R. was partially supported by the Applied Mathematics Program of the U.S. DOE Office of Science Advanced Scientific Computing Research under Contract No. DEAC02-05CH11231. L. M. was partially supported by the National Science Foundation under Grants No. DMR2011754 and No. DMR-1922321.

N. J. D. and D. C. F. contributed equally to this work.
* Corresponding author.

Lmahadev@g.harvard.edu

[1] V. Fleury, J.-F. Gouyet, and M. Lonetti, Branching in Nature: Dynamics and Morphogenesis of Branching Structures, from Cell to River Networks, Centre de Physique des Houches Vol. 14 (Springer, Berlin, 2001).

[2] P. Szymczak and A. J. Ladd, Geophys. Res. Lett. 38, 1 (2011).

[3] P. Grodzki and P. Szymczak, Phys. Rev. E 100, 033108 (2019).

[4] P. M. Duxbury, P. L. Leath, and P. D. Beale, Phys. Rev. B 36, 367 (1987).

[5] S. Zapperi, P. Ray, H. E. Stanley, and A. Vespignani, Phys. Rev. Lett. 78, 1408 (1997).

[6] P. Cerasi and P. Mills, Phys. Rev. E 58, 6051 (1998).

[7] N. Schorghofer, B. Jensen, A. Kudrolli, and D. H. Rothman, J. Fluid Mech. 503, 357 (2004).

[8] A. E. Lobkovsky, A. V. Orpe, R. Molloy, A. Kudrolli, and D. H. Rothman, J. Fluid Mech. 605, 47 (2008).

[9] D. McKenzie, J. Petrol. 25, 713 (1984).

[10] M. Spiegelman, J. Fluid Mech. 247, 17 (1993).

[11] M. Spiegelman, P. B. Kelemen, and E. Aharonov, J. Geophys. Res. Solid Earth 106, 2061 (2001).

[12] I. J. Hewitt, J. Glaciol. 57, 302 (2011).

[13] S. Camazine, Self-Organization in Biological Systems (Princeton University Press, Princeton, NJ, 2001).

[14] S. A. Ocko and L. Mahadevan, Phys. Rev. Lett. 114, 134501 (2015).

[15] A. Tero, S. Takagi, T. Saigusa, K. Ito, D. P. Bebber, M. D. Fricker, K. Yumiki, R. Kobayashi, and T. Nakagaki, Science 327, 439 (2010).

[16] H. Ronellenfitsch and E. Katifori, Phys. Rev. Lett. 123, 248101 (2019).

[17] A. Khuong, J. Gautrais, A. Perna, C. Sbaï, M. Combe, P. Kuntz, C. Jost, and G. Theraulaz, Proc. Natl. Acad. Sci. U.S.A. 113, 1303 (2016).

[18] A. E. Scheidegger, The Physics of Flow through Porous Media, rev. ed. (Macmillan, New York, 1960).

[19] J. Bear, Dynamics of Fluids in Porous Media (Dover, New York, 1988), p. 764.

[20] D. Drew and S. Passman, Theory of Multicomponent Fluids, Applied Mathematical Sciences Vol. 135 (Springer, New York, 1999).

[21] A. Mahadevan, A. V. Orpe, A. Kudrolli, and L. Mahadevan, Europhys. Lett. 98, 58003 (2012).

[22] See Supplemental Material at http://link.aps.org/ supplemental/10.1103/PhysRevLett.125.158002 for derivations, elaborations, descriptions of the numerical solution procedure, and visualizations of the dynamics of arborization shown in Figs. 3 and 4.

[23] G. I. Barenblatt, M. Bertsch, and C. Nitsch, Commun. Appl. Math. Comput. Sci. 1, 143 (2006).

[24] M. T. Heath, Scientific Computing: An Introductory Survey, McGraw-Hill Series in Computer Science (McGraw-Hill, New York, 1997). 